Pacific Journal of Mathematics

REAL PARTS OF UNIFORM ALGEBRAS 


\title{
REAL PARTS OF UNIFORM ALGEBRAS
}

\author{
JOHN M. F. O'CONNELL
}

\begin{abstract}
This paper is concerned with identifying those uniform algebras $B$ on $\Gamma=\{z:|z|=1\}$ for which $\operatorname{Re} B$-the space of real parts of the functions in $B$-equals $\operatorname{Re} A$, where $A$ denotes the disk algebra. It is shown that for any such algebra, there is an absolutely continuous homeomorphism $\Phi$ of $\Gamma$ onto $\Gamma$ so that $B=A(\Phi)=\{f(\Phi): f \in A\}$. A partial converse to this theorem also holds: If $\Phi$ is a homeomorphism of $\Gamma$ onto itself which is of class $C^{2}$ with nowhere vanishing derivative, then $\operatorname{Re} A(\Phi)=\operatorname{Re} A$.
\end{abstract}

For completeness, we recall that a uniform algebra on $\Gamma$ is defined as a subalgebra of $C(\Gamma)$ which is closed in the norm $\|f\|=\max _{\Gamma}|f|$, contains the constants and separates the points of $\Gamma$. The disk algebra is the particular uniform algebra consisting of all functions in $C(\Gamma)$ which extend continuously to $\{z:|z| \leqq 1\}$ to be analytic on $D=\{z:|z|<1\}$. Note that if $\Phi$ is a homeomorphism of $\Gamma$ onto itself, then $A(\Phi)$ is a uniform algebra. In this paper we will frequently use the convention of writing $f(\theta)$ for $f\left(e^{i \theta}\right)$ when $f$ belongs to $C(\Gamma)$.

The first result along the lines discussed in this paper is due to Hoffman and Wermer [7]. They prove that if $B$ is a uniform algebra on a compact Hausdorff space $X$ such that $\operatorname{Re} B$ is closed in the norm of uniform convergence, then $B=C(X)$; in particular, the theorem holds if $\operatorname{Re} B=C_{r}(X)$. A generalization of this fact by Sidney and Stout [10] says that if $K$ is a closed subset of $X$ and $\operatorname{Re} B_{K}$ is uniformly closed, then $B_{K}=C(K)$. Bernard [1, 2,3] has provided extensions in a different direction. He shows that a Banach algebra $B \cong C(X)$, with any norm, for which $\operatorname{Re} B$ is closed under uniform convergence must equal $C(X)$. Further, he gives some sufficient conditions on two Banach algebras $B_{1} \subseteq B_{2} \subseteq C(X)$, with $\operatorname{Re} B_{1}=\operatorname{Re} B_{2}$, to conclude that $B_{1}=B_{2}$.

The theorems in this paper have previously been announced in Abstracts 71T-B10 and 71T-B94 of the Notices of the American Mathematical Society, 18 (1971). The author wishes to express his appreciation to Professor John Wermer for his many helpful suggestions and conversations during the research which led to these results.

II. Algebras on $\Gamma$ with the same real parts.

Theorem 1. Let $B$ be a uniform algebra on $\Gamma$ with $\operatorname{Re} B=\operatorname{Re} A$. Then there exists a homeomorphism $\Phi$ of $\Gamma$ onto $\Gamma$ so that $B=A(\Phi)$. 
Proof. (1). Since $\cos (\theta)$ belongs to $\operatorname{Re} B$, there exists $v(\theta)$ so that $\psi(\theta)=\cos (\theta)+i v(\theta)$ belongs to $B . \quad \operatorname{Re} \psi(\theta)$ is one-to-one and decreasing on $[0, \pi]$ and one-to-one increasing on $[-\pi, 0]$; hence $C-$ $\psi(\Gamma)$ has a bounded component, or else $\psi(\Gamma)$ defines a single curve traced twice, once in each direction. In the latter case, $\psi(\Gamma)$ is a Jordan arc; it then follows by Mergelyan's theorem that the function $\operatorname{Re} z$ is a uniform limit of polynomials on $\psi(\Gamma)$ and we can conclude that $\operatorname{Re} \psi(\theta)=\cos (\theta) \in B$. Similarly there exists $u(\theta)$ in $\operatorname{Re} B$ so that $\psi_{1}(\theta)=u(\theta)+i \sin (\theta)$ belongs to $B$; and either $C-\psi_{1}(\Gamma)$ has bounded component or else $\sin (\theta) \in B$.

If both $\cos (\theta)$ and $\sin (\theta)$ are in $B$, then $B=C(\Gamma)$ and $\operatorname{Re} B \neq$ $\operatorname{Re} A$. Thus at least one of $C-\psi(\Gamma)$ and $C-\psi_{1}(\Gamma)$ has a bounded component. We will assume that $C-\psi(\Gamma)$ has a bounded component, which we call $W$. The remainder of the proof using $\psi_{1}$ involves only minor changes.

(2). The region $W$ is bounded by two ares on $\psi(\Gamma)$. Precisely, there are values $\theta_{1}$ and $\theta_{2}$ with $0 \leqq \theta_{1}<\theta_{2} \leqq \pi$ for which $\psi\left(\theta_{i}\right)=$ $\psi\left(-\theta_{i}\right), i=1,2$ and $\partial W=\psi\left(\left[\theta_{1}, \theta_{2}\right]\right) \cup \psi\left(\left[-\theta_{2},-\theta_{1}\right]\right)$. For $\theta$ not contained in $\left[\theta_{1}, \theta_{2}\right] \cup\left[-\theta_{2},-\theta_{1}\right], \operatorname{Re} \psi(\theta)<\operatorname{Re} \psi\left(\theta_{2}\right)$ or $\operatorname{Re} \psi(\theta)>\operatorname{Re} \psi\left(\theta_{1}\right)$.

Let $\tau$ be the Riemann map of $W$ onto $D$; $\tau$ extends continuously to $\bar{W}$, mapping $\partial W$ onto $\Gamma$ in a one-to-one fashion. For convenience we may suppose $(\tau \circ \psi)\left(\theta_{1}\right)=1$. We further extend $\tau$ by setting $\tau(z)=$ $(\tau \circ \psi)\left(\theta_{1}\right)$ for $z$ with $\operatorname{Re} z>\operatorname{Re} \psi\left(\theta_{1}\right)$ and $\tau(z)=(\tau \circ \psi)\left(\theta_{2}\right)$ for $z$ with $\operatorname{Re} z<\operatorname{Re} \psi\left(\theta_{2}\right)$. Then, letting $K$ denote $\psi(\Gamma)$ together with all its bounded complementary components, $\tau$ is continuous on $K$ and is analytic on its interior. Appealing again to Mergelyn's theorem, we conclude that $\tau$ is the uniform limit on $K$ of polynomials. Hence $\tau \circ \psi$ belongs to $B$. Put $\Phi=\tau \circ \psi$. Then $\Phi$ maps $\Gamma$ onto $\Gamma$ in the following manner: (see figure)
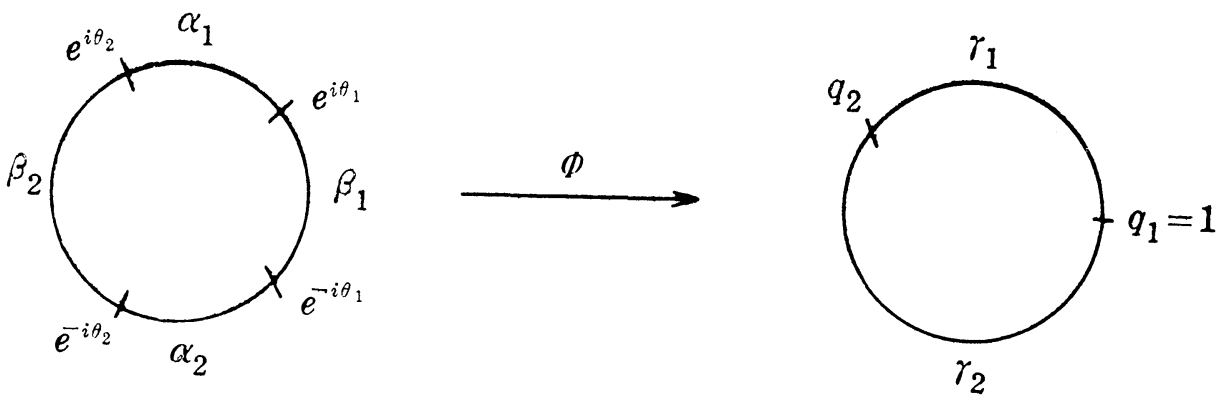

FIGURE

$\Phi$ takes the open ares $\alpha_{1}$ and $\alpha_{2}$ homeomorphically onto $\gamma_{1}$ and $\gamma_{2}$, and the closed ares $\beta_{1}$ and $\beta_{2}$ onto the points $q_{1}=\Phi\left(\theta_{1}\right)=1, q_{2}=\Phi\left(\theta_{2}\right)$ respectively. 
(3) For each measure $\nu$ on $\Gamma$ we define a measure $\nu^{*}$ on $\Gamma$ by $\nu^{*}(E)=\nu\left(\Phi^{-1}(E)\right)$, and for $g$ in $B$ and $\lambda$ on $\gamma_{1} \cup \gamma_{2}$ we put $g^{*}(\lambda)=$ $g\left(\Phi^{-1}(\lambda)\right)$. Let $\mu$ be a measure on $\Gamma$ with $\mu \perp B$ and $\mu^{*} \neq 0$. Such measures must exist as otherwise every $\mu \perp B$ would be the zero measure on $\alpha_{1} \cup \alpha_{2}$. Then for every closed subarc $E$ of $\alpha_{1} \cup \alpha_{2}$ of positive linear measure, it would follow that $\left.B\right|_{E}=C(E)$. Hence $\left.\operatorname{Re} A\right|_{E}=C_{R}(E)$ which is impossible.

If $g$ belongs to $B$ and $|z| \neq 1$, define

$$
T(g, z)=\int_{|\lambda|=1} \frac{(g d \mu)^{*}}{\lambda-z}(\lambda) .
$$

The function $T$ is analytic on $D$ and also on $\{z:|z|>1\}$. We claim that $T(g, z)=0$ if $|z|>1$. For, on that set,

$$
T(g, z)=\sum_{n=0}^{\infty} \frac{-1}{z^{n+1}} \int_{|\lambda|=1} \lambda^{n}(g d \mu) *(\lambda) .
$$

The term for $n=0$ is $\int_{|\lambda|=1}(g d \mu)^{*}=\int_{\Gamma} g d \mu=0$.

For each $n>1$,

$$
\begin{aligned}
\int_{|\lambda|=1} \lambda^{n}(g d \mu)^{*}= & \int_{\gamma_{1} \cup \gamma_{2}} \lambda^{n} g^{*}(\lambda) d \mu \mu^{*}(\lambda) \\
& +q_{1}^{n} \int_{\beta_{1}} g d \mu+q_{2}^{n} \int_{\beta_{2}} g d \mu \\
= & \int_{\alpha_{1} \cup \alpha_{2}} \Phi^{n}(t) g(t) d \mu(t) \\
& +\int_{\beta_{1}} q_{1}^{n} g(t) d \mu(t)+\int_{\beta_{2}} q_{2}^{n} g(t) d \mu(t) \\
= & \int_{|t|=1} \Phi^{n}(t) g(t) d \mu(t) \\
= & 0, \text { since } \Phi^{n} g \text { belongs to } B .
\end{aligned}
$$

(4) Let $\nu$ be any measure on $\Gamma$. Put $h(\theta)=$ the $\nu$-measure of the arc $\overparen{1 e^{i \theta}}$. At any point $\lambda_{0}=e^{i \theta_{0}}$ where $d h / d \theta\left(\theta_{0}\right)$ exists, we set $d \nu / d \lambda\left(\lambda_{0}\right)=d h / d \theta\left(\theta_{0}\right)$. Then for almost all values of $\lambda_{0}$ on $\Gamma$, we have

$$
\lim _{r e^{i \theta} \rightarrow \lambda^{\lambda_{0}}}\left[\int_{|\lambda|=1} \frac{d \nu(\lambda)}{\lambda-r e^{i \theta}}-\int_{|\lambda|=1} \frac{d \nu(\lambda)}{\lambda-\frac{1}{r} e^{i \theta}}\right]=2 \pi i \frac{d \nu}{d \lambda}\left(\lambda_{0}\right) .
$$

The limit is taken along non-tangential curves lying within the unit disk. The statement follows from Fatou's theorem by rewriting the terms inside the brackets as a Poisson integral. 
By applying this equality to the measures $(g d \mu)^{*}$ and using that $T(g, z)=0$ for $|z|>1$ we conclude that, for almost all $\lambda_{0}$ on $\gamma_{1} \cup \gamma_{2}$,

$$
\begin{aligned}
\lim _{\substack{z \rightarrow \lambda_{0} \\
|a|<i}} T(g, z) & =\lim _{\substack{z \rightarrow \lambda_{0} \\
|z|<1}} \int_{|\lambda|=1} \frac{(g d \mu)^{*}}{\lambda-z}(\lambda) \\
& =2 \pi i g^{*}\left(\lambda_{0}\right) \frac{d \mu^{*}}{d \lambda}\left(\lambda_{0}\right) .
\end{aligned}
$$

The function $T(1, z)$ is the Cauchy transform of the nonzero measure $\mu^{*}$. Since the Cauchy transform of a measure is zero almost everywhere- $d x d y$ if and only if the measure itself is zero ${ }^{1}$, and since $T(1, z)$ vanishes identically for $|z|>1$ it follows that $T(1, z)$ cannot vanish identically on $D$. Also, $T(1, z)$ has the boundary value $2 \pi i\left(d \mu^{*} / d \lambda\right)\left(\lambda_{0}\right)$ for almost all $\lambda_{0}$ on $\gamma_{1} \cup \gamma_{2}$. (Here boundary value means the limiting value along non-tangential curves approaching $\lambda_{0}$ from inside the unit disk). It is a theorem of Privalov and Lusin' that a function which is analytic on $D$ and which has the non-tangential limiting value zero on a set of positive measure on $\Gamma$ must vanish identically on $D$. Applying this result to $T(1, z)$ we can conclude that $d \mu^{*} / d \lambda\left(\lambda_{0}\right) \neq 0$ for almost all $\lambda_{0}$ on $\gamma_{1} \cup \gamma_{2}$.

For each $g$ contained in $B, T(g, z)$ is analytic on $D$ and has the boundary values $2 \pi \mathrm{i} g^{*}\left(\lambda_{0}\right) d \mu^{*} / d \lambda\left(\lambda_{0}\right)$ almost everywhere on $\gamma_{1} \cup \gamma_{2}$. Consequently, the function $G(z)=T(g, z) / T(1, z)$ is meromorphic on $D$ and has the boundary values $g^{*}\left(\lambda_{0}\right)$ almost everywhere on $\gamma_{1} \cup \gamma_{2}$.

(5) Now we show that each function $G(z)$ obtained in the manner just described is analytic on $D$. To this end, take $z_{0}$ in $D$ so that $T\left(1, z_{0}\right) \neq 0$. The functional $S$ defined on $B$ by $S(g)=G\left(z_{0}\right)$ is linear. For any two functions $g_{1}$ and $g_{2}$ belonging to $B$, the function $t(z)=$ $T(1, z) T\left(g_{1} g_{2}, z\right)-T\left(g_{1}, z\right) T\left(g_{2}, z\right)$ is analytic on $D$ and has zero boundary values almost everywhere. Hence, by the theorem Privalov and Lusin cited in the last paragraph, $t \equiv 0$ and the meromorphic functions

$$
\frac{T\left(g_{1} g_{2}, z\right)}{T(1, z)} \text { and } \frac{T\left(g_{1}, z\right)}{T(1, z)} \frac{T\left(g_{2}, z\right)}{T(1, z)}
$$

are identically equal whenever defined. Thus $S$ is a multiplicative linear functional on the commutative Banach algebra $B$ and as such has norm one. That is, $\left|G\left(z_{0}\right)\right| \leqq\|g\|$ for every $z_{0}$ with $T\left(1, z_{0}\right) \neq 0$. As the zeros of $T(1, z)$ form a discrete set, all the singularities of $G$ are removable and $G$ extends analytically to $D$. Furthermore, $G$ is bounded on $D$ with $|G(z)| \leqq\|g\|$ for all $z$ contained in $D$.

1 See Browder [4] or Gamelin [5] for details.

2 See Goluzin [6], p. 428, or Privalov [9]. 
(6). The next step is to show $G$ belongs to $A$. $G$ can be defined from $g^{*}$ using the Poisson integral formula. A well-known property of that formula says that $G$ has the nontangential boundary value $g^{*}\left(\lambda_{0}\right)$ at every point of continuity of $g^{*}$. Thus $G$ has the boundary values $g^{*}$ everywhere on $\gamma_{1} \cup \gamma_{2}$. The following result may be used to prove that $G$ has continuous boundary values everywhere on $\Gamma$.

THEOREM. $^{3}$ If $f(z) \rightarrow a$ as $z \rightarrow \infty$ along a straight line and $f(z) \rightarrow$ $b$ as $z \rightarrow \infty$ along another straight line, and $f(z)$ is regular and bounded in the angle between, then $a=b$ and $f(z) \rightarrow a$ uniformly in the angle.

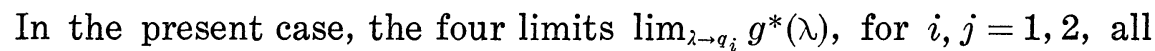
exist, because of the continuity of the function $g$. By considering neighborhoods of $q_{1}$ and $q_{2}$ in $\bar{D}$, mapping them to the upper half plane sending $q_{1}$ and $q_{2}$ in turn to the point at $\infty$, we can conclude that $\lim _{z \rightarrow q_{1}} G(z)$ and $\lim _{z \rightarrow q_{2}} G(z)$ both exist and that $G \in A$. In fact, we must have that $\lim _{z \rightarrow q_{1}} G(z)=g\left(\theta_{1}\right)=g\left(-\theta_{1}\right)$ and $\lim _{z \rightarrow q_{2}} G(z)=g\left({ }_{2}\right)=$ $g\left(-\theta_{2}\right)$, for every function $g$ belonging to $B$. But as $B$ separates points on $\Gamma$, this is impossible unless $\theta_{1}=0, \theta_{2}=\pi$. There, $\Phi$ is a homeomorphism of $\Gamma$ onto $\Gamma$.

( 7 ). Since $\Phi$ belongs to $B, A(\Phi) \subseteq B$ or equivalently $A \subseteq B\left(\Phi^{-1}\right)$. As $\operatorname{Re} B=\operatorname{Re} A \neq C_{R}(\Gamma)$, then $B\left(\Phi^{-1}\right) \neq C(\Gamma)$. Hence by Wermer's Maximality Theorem, $B\left(\Phi^{-1}\right)=A$ or $B=A(\Phi)$.

THEOREM 2. Let $\Phi$ be a homeomorphism of $\Gamma$ onto $\Gamma$ satisfying $\operatorname{Re} A(\Phi)=\operatorname{Re} A$. Then $\Phi$ is absolutely continuous.

Proof. We also have that $\operatorname{Re} A\left(\Phi^{-1}\right)=\operatorname{Re} A$. Let $m$ denote Lebesgue measure on $\Gamma$. We claim that if $K$ is a closed set with $m(K)=0$, then $m(\Phi(K))=0$. For, let $g \in C(\Phi(K))$. Then $\left.g \circ \Phi\right|_{K} \in C(K)$. Since we assume that $K$ is closed with $m(K)=0$, it follows that $\left.A\right|_{K}=C(K)^{4}$, so that there exists $G$ belonging to $A$ with $\left.G\right|_{K}=\left.g \circ \Phi\right|_{K}$. Thus $\left.g\right|_{\Phi_{(K)}}=\left.G \circ \Phi^{-1}\right|_{(K)}$. In particular, if $g \in C_{R}(\Phi(K))$, then $g \in$ $\left.\operatorname{Re} A\left(\Phi^{-1}\right)\right|_{\Phi(K)}=\left.\operatorname{Re} A\right|_{\Phi(K)}$. Hence $\left.\operatorname{Re} A\right|_{\mathscr{\Phi}(K)}$ is closed in the uniform norm. This implies that $\left.\left.\left.A\right|_{\Phi_{(K)}}=C(\Phi) K\right)\right)^{5}$. If $m(\Phi(K))>0, A$ would necessarily contain a nonzero function which vanished on a set of positive measure on $\Gamma$. This is impossible since the functions in $A$ satisfy the Jensen inequality $\log |f(0)| \leqq 1 / 2 \pi \int_{\Gamma} \log |f| d \theta$. Hence we must have $m(\Phi(K))=0$.

3 Titchmarsh [11], 5,64.

4 This is a theorem of Rudin and Carleson. See [5] for details.

5 This result is due to Sidney and Stout [10]. 
Now if we define a measure $\mu$ on $\Gamma$ by $\mu(E)=m(\Phi(E))$ we have that $\mu$ is absolutely continuous with respect to $m$. To see this, let $m(E)=0$. There exists an $F_{o}$ set $F \subseteq E$ such that $\mu(E \backslash F)=0$. We can write $F=\bigcup_{n=1}^{\infty} K_{n}$ where $K_{n}$ is closed and $K_{n} \subseteq K_{n+1}$ for each $n$. From the last paragraph, it follows that $\mu\left(K_{n}\right)=0$. Hence $\mu(F)=0$ and $m(\phi(E))=\mu(E)=0$.

For an arc $\widehat{\alpha \beta}, \mu(\widetilde{\alpha \beta})=|\Phi(\beta)-\Phi(\alpha)|$. The absolute continuity of $\mu$ implies that for $\varepsilon>0$, there is a $\delta>0$ so that $m(E)<\delta \Rightarrow$ $\mu(E)<\varepsilon$. Hence if $\widetilde{\alpha_{i} \beta_{i}}, 1 \leqq i \leqq N$, are disjoint $\operatorname{arcs}$ with $\sum_{i=1}^{N} m\left(\widetilde{\alpha_{i} \beta_{i}}\right)<$ $\delta$ then $\sum_{i=1}^{N}\left|\Phi\left(\beta_{i}\right)-\Phi\left(\alpha_{i}\right)\right|<\varepsilon$. Therefore $\Phi$ is absolutely continuous.

REMARKS. (1) Theorem 1 cannot be extended freely: there exist examples of sets $X$, and two uniform algebras $A$ and $B$ defined on $X$ satisfying $\operatorname{Re} A=\operatorname{Re} B$, but for which no homeomorphism $\Phi$ yields $B=A(\Phi)$.

(2) If, on $\Gamma, B=\bar{A}$, the set of complex conjugates of the functions in $A$, the map $z \rightarrow \bar{z}$ is a suitable choice for $\Phi$.

III. Sufficient conditions on $\Phi$ to conclude $\operatorname{Re} A(\Phi)=\operatorname{Re} A$.

Definition. Let $\operatorname{Re} A^{\dagger}=\{f: f$ is continuous on $\boldsymbol{R}$, with period $2 \pi$, and so that the function $F$, defined on $\Gamma$ by $F\left(e^{i \theta}\right)=f(\theta)$, belongs to $\operatorname{Re} A$.

Definition. For $u$ in $\operatorname{Re} A$, define \|\|$u\|=\| u\|+\| \tilde{u} \|$ where $\|\cdot\|$ is the uniform norm on $\Gamma, u+i \widetilde{u}$ belongs to $A$ and $\widetilde{u}(0)=0$. For $f$ in $(\operatorname{Re} A)^{\dagger}, \widetilde{f}$ denotes the function in $(\operatorname{Re} A)^{\dagger}$ such that $G\left(e^{i \theta}\right)=f(\theta)+$ $i \widetilde{f}(\theta)$ belongs to $A$ and $\operatorname{Im} G(0)=0$. We put $\|f\|=\max _{\boldsymbol{R}}|f|$ and

$$
\|\mid f\|=\|f\|+\|\tilde{f}\| \text {. }
$$

With $\|\cdot \cdot\|$ as a norm, $\operatorname{Re} A$ and $(\operatorname{Re} A)^{\dagger}$ have the structure of real Banach spaces in which smooth functions are dense.

Lemma 1. Suppose $\phi$ is a homeomorphism of $[-\pi, \pi]$ onto $[\alpha$, $2 \pi+\alpha]$, with inverse $\psi$, and satisfying

(i) $\phi(0)=0$,

(ii) $\phi$ is of class $C^{2}, \phi^{\prime}(-\pi)=\phi^{\prime}(\pi), \psi^{\prime}(\alpha)=\psi^{\prime}(\alpha+2 \pi)$,

(iii) $\left|\phi^{\prime}(t)\right| \geqq \gamma>0,\left|\psi^{\prime}(t)\right| \geqq \delta>0$ and $\left|\psi^{\prime \prime}(t)\right| \leqq M$ for all $t$ in $[-\pi, \pi]$.

Then, for a smooth function $f$ in $(\operatorname{Re} A)^{\dagger}, f \circ \phi$ which is defined on $[-\pi, \pi]$, if extended periodically to $\boldsymbol{R}$, lies in $(\operatorname{Re} A)^{\dagger}$. There exists a constant $C>0$, depending only on $\gamma, \delta$ and $M$, so that

$$
|\widetilde{f \circ \phi}(0)-\tilde{f}(0)| \leqq C|| f \| .
$$


Consequently,

$$
|\widetilde{f \circ \phi}(0)| \leqq(C+1)|||f|||
$$

Proof. We can assume, with no loss of generality, that $\phi^{\prime}(t) \geqq \gamma$ on $[-\pi, \pi]$. The proof if $\phi$ is a decreasing function is almost identical. Our assumption means that $\phi(-\pi)=\alpha<0$.

Let $f$ be a smooth function in $(\operatorname{Re} A)^{\dagger}$. As $\phi(-\pi)+2 \pi=\phi(\pi)$, then $f \circ \phi$ can be extended to $R$, continuously with period $2 \pi$. Since $f$ and $\phi$ are smooth and $\phi^{\prime}(-\pi)=\phi^{\prime}(\pi), f \circ \phi$ is smooth and thus belongs to $(\operatorname{Re} A)^{\dagger}$.

Assume first that $f(0)=0$. The integral formula for evaluating harmonic conjugates gives ${ }^{6}$

$$
\begin{aligned}
\tilde{f}(0) & =-\frac{1}{2 \pi} \int_{0}^{\pi} \frac{f(t)-f(-t)}{\tan \frac{t}{2}} d t \\
& =-\frac{1}{2 \pi} \int_{-\pi}^{\pi} \frac{f(t)}{\tan \frac{t}{2}} d t \\
& =-\frac{1}{2 \pi} \int_{\alpha}^{2 \pi+\alpha} \frac{f(t)}{\tan \frac{t}{2}} d t .
\end{aligned}
$$

$$
\text { Also } \begin{aligned}
\widetilde{f \circ \phi}(0)= & -\frac{1}{2 \pi} \int_{0}^{\pi} \frac{(f \circ \phi)(t)-(f \circ \phi)(-t)}{\tan \frac{t}{2}} d t \\
= & -\frac{1}{2 \pi} \int_{0}^{\pi} \frac{\left(f \circ \phi^{\prime}\right)(t)}{\tan \frac{t}{2}} d t-\frac{1}{2 \pi} \int_{-\pi}^{0} \frac{(f \circ \phi)(t)}{\tan \frac{t}{2}} d t \\
= & -\frac{1}{2 \pi} \int_{0}^{2 \pi+\alpha} \frac{f(u)}{\tan \frac{\psi(u)}{2}} \psi^{\prime}(u) d u \\
& -\frac{1}{2 \pi} \int_{\alpha}^{0} \frac{f(u)}{\tan \frac{\psi(u)}{2} \psi^{\prime}(u) d u} \\
= & -\frac{1}{2 \pi} \int_{\alpha}^{2-+\alpha} \frac{f(u) \psi^{\prime}(u)}{\tan \frac{\psi(u)}{2}} d u .
\end{aligned}
$$

All these integrals are absolutely convergent because of the smoothness of $f$ and $\phi$, and because $f(0)=\phi(0)=0$. We use the fact that $x \cot x$ has a series expansion 


$$
x \cot x=\sum_{n=0}^{\infty} A_{n} x^{n}
$$

which converges for $|x|<\pi$. Hence

$$
\frac{1}{\tan x}=\frac{1}{x}+a(2 x)
$$

where $a(t)$ is real analytic for $|t|<2 \pi$. Substitution of (3) into (1) and (2) yields

$$
\tilde{f}(0)=-\frac{1}{2 \pi} \int_{\alpha}^{2 \pi+\alpha} f(t)\left[\frac{1}{\frac{t}{2}}+a(t)\right] d t
$$

and

$$
\widetilde{f \circ \phi}(0)=-\frac{1}{2 \pi} \int_{\alpha}^{2 \pi+\alpha} f(t)\left[\frac{1}{\frac{\psi(t)}{2}}+a(\psi(t))\right] \psi^{\prime}(t) d t .
$$

Since $-2 \pi<\alpha<0, a(t)$ is continuous on $[\alpha, 2 \pi+\alpha]$, and since $-\pi \leqq$ $\psi(t) \leqq \pi$, these two integrals are absolutely convergent. Subtracting them, we get

$$
\begin{aligned}
|\widetilde{f \circ \phi(0)}-\widetilde{f}(0)| \leqq & \frac{1}{2 \pi}\|f\| \int_{\alpha}^{2 \pi+\alpha}\left\{2\left|\frac{\psi^{\prime}(t)}{\psi(t)}-\frac{1}{t}\right|\right. \\
& \left.+|a(\psi(t))| \psi^{\prime}(t)+|a(t)|\right\} d t .
\end{aligned}
$$

We estimate each term in the integrand. For the first term, we write $\psi(t)=t \psi_{1}(t) . \quad \psi_{1}$ is smooth on $[\alpha, 2 \pi+\alpha]$ and $\psi_{1}(0) \neq 0$. For each $t$ in $[\alpha, 2 \pi+\alpha]$,

$$
\begin{aligned}
\psi(t) & =\int_{0}^{1} \frac{d}{d s} \psi(s t) d s \\
& =\int_{0}^{1} t \psi^{\prime}(s t) d s \\
& =t \int_{0}^{1} \psi^{\prime}(s t) d s .
\end{aligned}
$$

Thus

$$
\psi_{1}(t)=\int_{0}^{1} \psi^{\prime}(s t) d s \geqq \delta
$$

Also, 


$$
\begin{gathered}
\psi_{1}^{\prime}(t)=\int_{0}^{1} \frac{d}{d t} \psi^{\prime}(s t) d s \\
=\int_{0}^{1} s \psi^{\prime \prime}(s t) d s \\
\Longrightarrow\left|\psi_{1}^{\prime}(t)\right| \leqq M \int_{0}^{1} s d s=\frac{M}{2} \\
\Longrightarrow\left|\frac{\psi^{\prime}(t)}{\psi(t)}-\frac{1}{t}\right|=\left|\frac{\psi_{1}^{\prime}(t)}{\psi_{1}(t)}\right| \leqq \frac{M}{2 \delta} \\
\Longrightarrow \int_{\alpha}^{2 \pi+\alpha}\left|\frac{\psi^{\prime}(t)}{\psi^{\prime}(t)}-\frac{1}{t}\right| d t \leqq \frac{M \pi}{\delta} .
\end{gathered}
$$

For the second term in (4),

$$
\int_{\alpha}^{2 \pi+\alpha}|| a(\psi(t))\left|\psi^{\prime}(t) d t=\int_{-\pi}^{\pi}\right| a(t) \mid d t .
$$

For the last term, we note that

$$
\begin{aligned}
2 \pi+\alpha & =\dot{\phi}(\pi) \\
& =\phi(\pi)-\phi(0) \\
& =\pi \phi^{\prime}(r) \text { for some } r \in[0, \pi] . \\
\Longrightarrow 2 \pi+\alpha & \geqq \gamma \pi \\
\Longrightarrow \alpha & \geqq \gamma \pi-2 \pi .
\end{aligned}
$$

Similarly,

$$
\begin{aligned}
-\alpha & =-\phi(-\pi) \\
& =\phi(0)-\phi(-\pi) \\
& =\pi \phi^{\prime}\left(r_{1}\right) \text { for some } r_{1} \in[-\pi, 0] . \\
\Longrightarrow-\alpha & \geqq \gamma \pi \\
\Longrightarrow 2 \pi+\alpha & \leqq 2 \pi-\gamma \pi .
\end{aligned}
$$

Thus,

$$
\int_{\alpha}^{2 \pi+\alpha}|a(t)| d t \leqq \int_{-2 \pi+\gamma \pi}^{2 \pi-\gamma \pi}|a(t)| d t .
$$

The inequalities (4), (5), (6) and (7) imply

$$
\begin{aligned}
|\widetilde{f \circ \phi}(0)-\widetilde{f}(0)| \leqq & \|f\| \frac{1}{2 \pi}\left[2 \pi \frac{M}{\delta}+\int_{-\pi}^{\pi}|a(t)| d t\right. \\
& \left.+\int_{-2 \pi+\gamma \pi}^{2 \pi-\gamma \pi}|a(t)| d t\right] .
\end{aligned}
$$

Put $C / 2$ equal to the coefficient of $\|f\|$ in (8). Note that $C$ depends only on $M, \delta$ and $\gamma$. That inequality applies to smooth functions in 
$(\operatorname{Re} A)^{\dagger}$ vanishing at $x=0$. Now, let $f$ be any smooth function in $(\operatorname{Re} A)^{\dagger}$. We can apply (8) to $f-f(0)$. Since $\left.\tilde{f}(0)=\overline{(f-f(0)}\right)(0)$ and $\widetilde{f \circ \phi}(0)=\widetilde{[(f-f(0)) \circ \phi]}(0)$, we conclude that

$$
\begin{aligned}
|\widetilde{f \circ \phi}(0)-\widetilde{f}(0)| & \leqq\|f-f(0)\| \frac{C}{2} \\
\Longrightarrow|\widetilde{f \circ \phi}(0)-\widetilde{f}(0)| & \leqq C\|f\| \\
\Longrightarrow|\widetilde{f \circ \phi}(0)| & \leqq C\|f\|+|\tilde{f}(0)| \\
& \leqq C\|f\|+\|\tilde{f}\| \\
& \leqq(C+1)\|\| f \| \mid .
\end{aligned}
$$

Definition. For $\beta>0$ define $C_{\beta}$ by

$C_{\beta}=\left\{\Phi: \Phi\right.$ is a homeomorphism of $\Gamma$ onto $\Gamma$ of class $C^{2}$ satisfying:

$$
\begin{aligned}
\beta \leqq & \left|\frac{d}{d t} \Phi\left(e^{i t}\right)\right| \leqq \frac{1}{\beta} \\
\beta \leqq & \left|\frac{d}{d t} \Phi^{-1}\left(e^{i t}\right)\right| \leqq \frac{1}{\beta} \\
& \left|\frac{d^{2}}{d t^{2}} \Phi\left(e^{i t}\right)\right| \leqq \frac{1}{\beta} \\
& \left.\left|\frac{d^{2}}{d t^{2}} \Phi^{-1}\left(e^{i t}\right)\right| \leqq \frac{1}{\beta}\right\} .
\end{aligned}
$$

Lemma 2. There exists $K_{\beta}>0$, depending only on $\beta$ such that for all $\Phi$ belonging to $C_{\beta}$ with $\Phi(1)=1$ and for all smooth functions $F$ in $R e A$, we have

$$
|\widetilde{F(\Phi)}(1)| \leqq K_{\beta}|||F|||
$$

Proof. Define $\phi$ on $[-\pi, \pi]$ by $\Phi\left(e^{i t}\right)=e^{i \phi(t)}$. Then $\phi$ is of class $C^{2}$ and $\phi(0)=0$. If $\Psi=\Phi^{-1}$ and $\Psi\left(e^{i u}\right)=e^{i \eta(u)}$ for $u \in \phi([-\pi, \pi])$, then $\psi$ is the inverse of $\phi$. Furthermore

$$
\begin{aligned}
& \left|\phi^{\prime}(t)\right|=\left|\frac{d}{d t} \Phi\left(e^{i t}\right)\right|, \\
& \left|\psi^{\prime}(t)\right|=\left|\frac{d}{d t} \Psi\left(e^{i t}\right)\right|,
\end{aligned}
$$

and

$$
\left|\psi^{\prime \prime}(t)\right|=\left|\frac{d^{2}}{d t^{2}} \Psi\left(e^{i t}\right)\right|+\left|\frac{d}{d t} \Psi\left({ }^{i t}\right)\right|^{2}
$$

If we set $\gamma=\beta, \delta=\beta$ and $M=1 / \beta+1 / \beta^{2}$, then $\phi$ satisfies the 
hypotheses of Lemma 1 , and if we apply the lemma to $\phi$, the constant $C$ obtained in (8) depends only on $\beta$.

If $F$ is a smooth function in $\operatorname{Re} A$ and if $f$ is defined on $R$ by $f(\theta)=F\left(e^{i \theta}\right)$, then $f$ is a smooth function in $(\operatorname{Re} A)^{\dagger}$ with $\|F\|=$ \|\|$f \|$ and $\widetilde{f \circ \phi}(0)=\widetilde{F \circ \Phi}(1)$. Hence (9) yields that

$$
|\widetilde{F(\Phi)}(1)| \leqq(C+1)|||F|||
$$

Therefore the lemma is proved by defining $K_{\beta}=C+1$.

Lemma 3. If $\Phi$ belongs to $C_{\beta}$, then

$$
|\widetilde{F(\Phi)}(1)| \leqq K_{\beta}|| F|| \mid
$$

for all smooth functions $F$ contained in $\operatorname{Re} A$.

Proof. Define $\lambda$ by $\Phi(1)=e^{i \lambda}$ and set $\Phi_{1}=e^{-i \lambda} \Phi$. Note that $\Phi_{1}(1)=1$. If $\Psi=\Phi^{-1}$ and $\Psi_{1}=\Phi_{1}^{-1}$ then $\Psi_{1}\left(e^{i t}\right)=\Psi\left(e^{i(t+\lambda)}\right)$. We check that $\Phi_{1} \in C_{\beta}$ by noting that

$$
\begin{aligned}
\left|\frac{d}{d t} \Phi_{1}\left(e^{i t}\right)\right| & =\left|\frac{d}{d t} \Phi\left(e^{i t}\right)\right| \\
\left|\frac{d^{2}}{d t^{2}} \Phi_{1}\left(e^{i t}\right)\right| & =\left|\frac{d^{2}}{d t^{2}} \Phi\left(e^{i t}\right)\right| \\
\left|\frac{d}{d u} \Psi_{1}\left(e^{i u}\right)\right| & =\left|\frac{d}{d u} \Psi\left(e^{i(u+\lambda)}\right)\right| \\
\left|\frac{d^{2}}{d u^{2}} \Psi_{1}\left(e^{i u}\right)\right| & =\left|\frac{d^{2}}{d u^{2}} \Psi\left(e^{i(u+\lambda)}\right)\right| .
\end{aligned}
$$

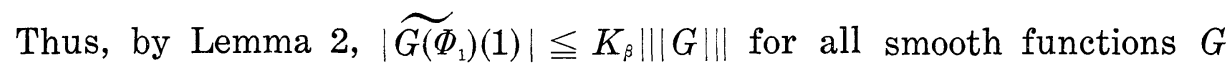
belonging to $\operatorname{Re} A$. Now, given $F$ smooth in $\operatorname{Re} A$, put $G\left(e^{i \theta}\right)=F\left(e^{i(\theta+\lambda)}\right)$. Then $G\left(\Phi_{1}\right)=F(\Phi), \widetilde{G\left(\Phi_{1}\right)}=\widetilde{F(\Phi)}$ and $\|F\|=\|G\|$. Hence

$$
|\widetilde{F(\Phi)}(1)| \leqq K_{\beta}|| F|| \mid
$$

Lemma 4. For $\Phi \in C_{\beta}$ and $\zeta$ with $|\zeta|=1$,

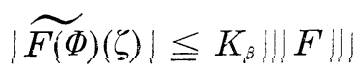

for all smooth functions $F$ in $\operatorname{Re} A$.

Proof. Fix $\zeta=e^{i \lambda}$ on $\Gamma$. Define $\Phi^{*}$ by $\Phi^{*}\left(e^{i \theta}\right)=\Phi\left(e^{\imath(\theta+\lambda)}\right)$. By comparing the derivatives of $\Phi, \Phi^{*}$ and their respective inverses in a manner similar to that in Lemma 3 , we can show that $\Phi^{*} \in C_{\beta}$. Therefore, for a smooth function $F$ in $\operatorname{Re} A$, 


$$
\left|\widetilde{F\left(\Phi^{*}\right)(1)}\right| \leqq K_{\beta}|| F|| \mid
$$

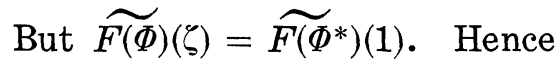

$$
|\widetilde{F(\Phi)}(\zeta)| \leqq K_{\beta}|| F|| \mid
$$

Lemma 5. If $\Phi$ belongs to $C_{\beta}$ for some $\beta>0$, then $\operatorname{Re} A(\Phi) \subseteq$ $\operatorname{Re} A$.

Proof. From Lemma 4, it follows that if $F$ is a smooth function in $\operatorname{Re} A$,

$$
\left\|\widetilde{F(\Phi)} \leqq K_{\beta}|\|F\||\right.
$$

Hence

$$
\begin{aligned}
|\|F(\Phi)\|| & =\|F(\Phi)\|+\|\widetilde{F(\Phi)}\| \\
& \leqq\|F\|+K_{\beta}\|F\|\left|\leqq\left(K_{\beta}+1\right)\|F \mid\| .\right.
\end{aligned}
$$

Defining $T$ by $T(F)=F(\Phi)$, we see that $T$ is a bounded linear transformation defined on smooth functions in $\operatorname{Re} A$ and mapping into $\operatorname{Re} A$. The domain of $T$ is dense in $\operatorname{Re} A$, so that $T$ has a norm preserving extension which maps $\operatorname{Re} A \rightarrow \operatorname{Re} A$; we also denote this extension by $T$. If $u$ belongs to $\operatorname{Re} A$, there are smooth function $F_{n}$ in $\operatorname{Re} A$ with $\left\|F_{n}-u\right\| \| \rightarrow 0$, and therefore $\left\|F_{n}(\Phi)-T u\right\| \mid \rightarrow 0$. These imply that $\left\|F_{n}-u\right\| \rightarrow 0$ and $\left\|F_{n}(\Phi)-T u\right\| \rightarrow 0$. Hence $T u=u(\Phi)$. That is $u(\Phi)$ belongs to $\operatorname{Re} A$ for every $u$ in $\operatorname{Re} A$.

Theorem 3. Let $\Phi$ belong to $C_{\beta}$ for some $\beta>0$. Then $\operatorname{Re} A(\Phi)=$ $\operatorname{Re} A$.

Proof. We apply Lemma 5 to $\Phi$ and $\Phi^{-1}$, both of which are contained in $C_{\beta}$, to conclude $\operatorname{Re} A(\Phi) \subseteq \operatorname{Re} A$ and $\operatorname{Re} A\left(\Phi^{-1}\right) \subseteq \operatorname{Re} A$. Therefore $\operatorname{Re} A(\Phi)=\operatorname{Re} A$.

Corollary. Let $\Phi$ be a homeomorphism of $\Gamma$ onto $\Gamma$ of class $C^{2}$ with $d / d t\left(\Phi\left(e^{i t}\right)\right) \neq 0$ for all $t$. Then $\operatorname{Re} A(\Phi)=\operatorname{Re} A$.

REMARK. The assumption that $d / d t\left(\Phi\left(e^{i t}\right) \neq 0\right.$ cannot be eliminated, as there do exist examples of homeomorphisms $\Phi$ of $\Gamma$ onto $\Gamma$ which are of class $C^{\infty}$, but for which $\operatorname{Re} A(\Phi) \neq \operatorname{Re} A$.

\section{REFERENCES}

1. A. Bernard, Une caracterisation de $C(X)$ parmi les algèbres de Banach, C. R. Acad. Sc. Paris, 267 (1968), 634-635, Series A. 
2. - Comparaison d'algèbres de fonctions à l'aide des parties reélles de leurs éléments, C. R. Acad. Sc. Paris, 270 (1970), 29-32, Series A.

3. - Algèbres ultraseparantes de fonctions continues, C. R. Acad. Sc. Paris, 270 (1970), 818-819, Series A.

4. A. Browder, Introduction to Function Algebras, W. A. Benjamin, Inc., 1969.

5. T. W. Gamelin, Uniform Algebras, Prentice-Hall, 1969.

6. G. M. Goluzin, Geometric Theory of Functions of a Complex Variable, Amerian Mathematical Society, Translations of Mathematical Monographs, Vol. 26, 1969.

7. K. Hoffman and J. Wermer, A Characterization of $C(X)$, Pacific Math., J. 12 (1962), 941-944.

8. Y. Katznelson, An Introduction to Harmonic Analysis, Wiley, 1968.

9. I. I. Privalov, Randeigenschaften analytischer Funktionem, VEB Deutscher Verlag, 1956.

10. S. J. Sidney and E. L. Stout, $A$ note on interpolation, Proc. Amer. Math. Soc., 19 (1968), 380-382.

11. E. Titchmarsh, The Theory of Functions, Oxford Univ. Press, 1939.

12. J. Wermer, Function rings on Riemann surfaces, Annals of Math., 67 (1958), 45-71.

13. A. Zygmund, Trigonometric Series 2nd. ed., Cambridge Univ. Press, 1959

Received July 23, 1971.

BROWN UNIVERSITY 



\section{PACIFIC JOURNAL OF MATHEMATICS}

\section{EDITORS}

D. Gilbarg and J. Milgram Stanford University

Stanford, California 94305

R. A. Beaumont

University of Washington

Seattle, Washington 98105
J. DugundJI

Department of Mathematics

University of Southern California

Los Angeles, California 90007

RICHARD ARENS

University of California

Los Angeles, California 90024

ASSOCIATE EDITORS
E. F. BECKENBACH
B. H. NeumanN
F. WOLF
K. YoSHIDA

\section{SUPPORTING INSTITUTIONS}

UNIVERSITY OF BRITISH COLUMBIA

CALIFORNIA INSTITUTE OF TECHNOLOGY

UNIVERSITY OF CALIFORNIA

MONTANA STATE UNIVERSITY

UNIVERSITY OF NEVADA

NEW MEXICO STATE UNIVERSITY

OREGON STATE UNIVERSITY

UNIVERSITY OF OREGON

OSAKA UNIVERSITY
UNIVERSITY OF SOUTHERN CALIFORNIA

STANFORD UNIVERSITY

UNIVERSITY OF TOKYO

UNIVERSITY OF UTAH

WASHINGTON STATE UNIVERSITY

UNIVERSITY OF WASHINGTON

$\stackrel{*}{*} \stackrel{*}{*} \stackrel{*}{*}$ AMERICAN MATHEMATICAL SOCIETY

NAVAL WEAPONS CENTER 


\section{Pacific Journal of Mathematics}

\section{Vol. 46, No. $1 \quad$ November, 1973}

Allan Francis Abrahamse, Uniform integrability of derivatives on $\sigma$-lattices .......................................... 1

Ronald Alter and K. K. Kubota, The diophantine equation $x^{2}+D=p^{n} \ldots \ldots \quad 11$

Grahame Bennett, Some inclusion theorems for sequence spaces .......... 17

William Cutler, On extending isotopies ........................ 31

Robert Jay Daverman, Factored codimension one cells in Euclidean

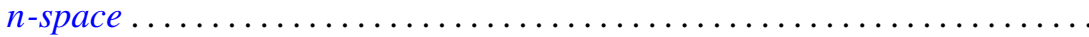

Patrick Barry Eberlein and Barrett O’Neill, Visibility manifolds ............ 45

M. Edelstein, Concerning dentability .......................... 111

Edward Graham Evans, Jr., Krull-Schmidt and cancellation over local

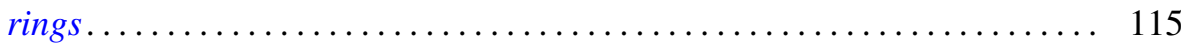

C. D. Feustel, A generalization of Kneser's conjecture ................ 123

Avner Friedman, Uniqueness for the Cauchy problem for degenerate parabolic equations .......................................... 131

David Golber, The cohomological description of a torus action ............ 149

Alain Goullet de Rugy, Un théorème du genre "Andô-Edwards" pour les

Fréchet ordonnés normaux..............................

Louise Hay, The class of recursively enumerable subsets of a recursively enumerable set ........................................

John Paul Helm, Albert Ronald da Silva Meyer and Paul Ruel Young, On orders of translations and enumerations...

Julien O. Hennefeld, A decomposition for $B(X)^{*}$ and unique Hahn-Banach

extensions

Gordon G. Johnson, Moment sequences in Hilbert space .

Thomas Rollin Kramer, A note on countably subparacompact spaces ...

Yves A. Lequain, Differential simplicity and extensions of a derivation ....

Peter Lorimer, A property of the groups Aut $\mathrm{PU}\left(3, q^{2}\right) \ldots$

225

Yasou Matsugu, The Levi problem for a product manifold.

231

John M.F. O'Connell, Real parts of uniform algebras ......

William Lindall Paschke, A factorable Banach algebra without bounded

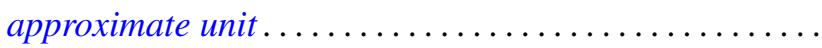

Ronald Joel Rudman, On the fundamental unit of a purely cubic field ....

Tsuan Wu Ting, Torsional rigidities in the elastic-plastic torsion of simply connected cylindrical bars .........................

Philip C. Tonne, Matrix representations for linear transformations on analytic sequences...................................

Jung-Hsien Tsai, On E-compact spaces and generalizations of perfect

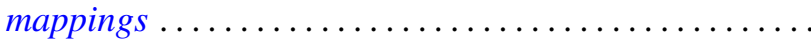

Alfons Van Daele, The upper envelope of invariant functionals majorized by an invariant weight. .. 